Document downloaded from:

http://hdl.handle.net/10251/63865

This paper must be cited as:

Báguena Albaladejo, M.; Tavares De Araujo Cesariny Calafate, CM.; Cano Escribá, JC.; Manzoni, P. (2015). An Adaptive Anycasting Solution for Crowd Sensing in Vehicular Environments. IEEE Transactions on Industrial Electronics. 62(12):7911-7919. doi:10.1109/TIE.2015.2447505.

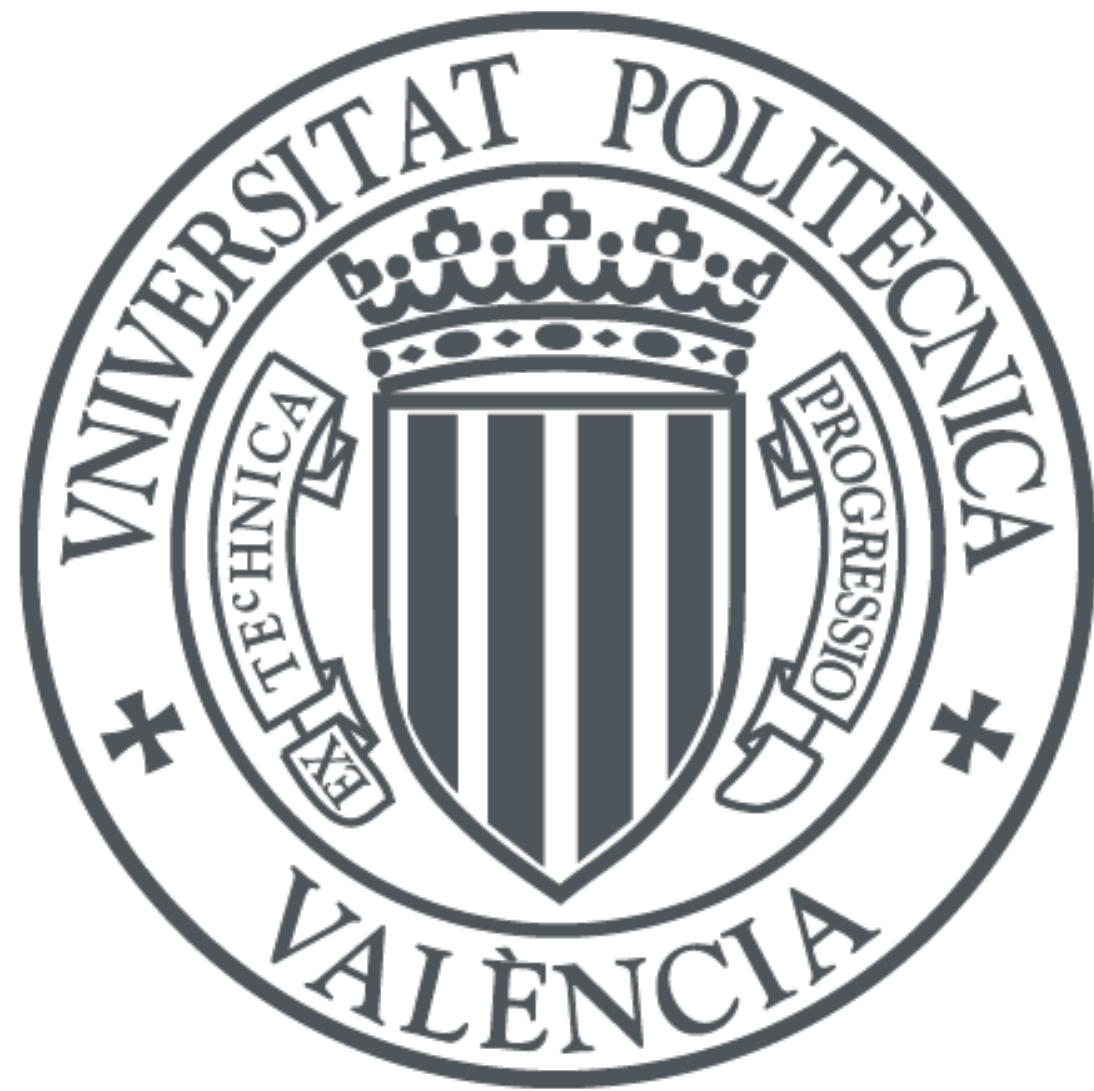

The final publication is available at

http://dx.doi.org/ 10.1109/TIE.2015.2447505

Copyright Institute of Electrical and Electronics Engineers (IEEE)

Additional Information

"C2015 IEEE. Personal use of this material is permitted. Permission from IEEE must be obtained for all other uses, in any current or future media, including reprinting/republishing this material for advertising or promotional purposes, creating new collective works, for resale or redistribution to servers or lists, or reuse of any copyrighted component of this work in other works." 


\section{An Adaptive Anycasting Solution for Crowd Sensing in Vehicular Environments}

\begin{abstract}
Vehicular networks can be seen as the new key enablers of the future networked society. Vehicles traveling can act as mobile sensors and collect a variety of information that can be used to enable various new services like environment monitoring, traffic management, urban surveillance, and so on.

In this paper we present AVE ("adaptive Anycasting solution for Vehicular Environments"), a message delivery protocol which combines geographical and topological information to dynamically adapt its behavior to network conditions. We focus on V2I connectivity for cloud services, where the vehicles send the sensed information as individual and independent messages to a cloud service in the Internet. This scenario requires the access to any available close-by Road Side Unit (RSU), thus making anycasting the ideal delivery mechanism.

Simulations results show that the hybrid and adaptive approach of AVE is able to improve network performance. For example, regarding delivery ratio, AVE outperforms DYMO by $10 \%$ in sparse scenarios and outperforms DTN techniques by $10 \%$ in dense scenarios.
\end{abstract}

Index Terms-vehicular networks; crowd sensing; adaptive; anycasting

\section{INTRODUCTION}

Vehicular networks (e.g., [1], [2], [3]) can be seen as the new key enablers of the future networked society. Vehicles traveling can act as mobile sensors and collect a variety of information. The vast information collected by vehicles can be used to enable various new services like environment monitoring, pollution measurements, safety, smart navigation, traffic management, and urban surveillance. The acquisition, sharing, processing, and transmission of data from vehicles foresee a new way to manage the communications.

Vehicular networks create very varying topologies along time, often including isolated nodes, variable quality links, and variable nodes densities. In the last years, there have been a significant number of messages delivery protocols for VANETs reported in the literature. For a complete review of the current status of the art in this area please refer to [4], [5]; in [6] the authors identify the open challenges in R\&D from both a scientific and an industrial point of view.

Anyway, such highly mutable and heterogeneous context represents a problem for network protocols, which are typically designed for more homogeneous scenarios: state-ofthe-art routing protocols designed for one environment either work poorly or are unsuitable in others; limitations have been demonstrated, see [7]. For example, protocols based on traditional link-state or distance vector routing cannot work in DTNs where a contemporaneous end-to-end path is unavailable. Most proactive as well as on-demand MANET protocols (e.g., [8], [9]) also assume the availability of a contemporaneous end-to-end path. Geographical routing (e.g., [10]), i.e., taking advantage of information about the location, emerged as a more efficient solution. However, geographical algorithms cannot achieve the best performance in every situation, the worst of which is related with the handling of "local minimum". Likewise, DTN protocols commonly use packet replication to reduce delays, but packet replication performs poorly in predictable, dense vehicular networks ([11]).

Focussing on vehicular sensing, anycasting is the ideal mechanism since it provides the transport of information towards intended receivers while meeting certain design objectives; in anycasting, the planned receivers are those specifying interest in the information. Users may also define arbitrary interests: "parking spots availability in the center of the town", "traffic status close to the stadium", etc. An optimal solution would involve low delay, high reliability, low memory occupancy, and low message passing overhead. See for example [12] or [13].

We therefore present AVE ("adaptive Anycasting solution for Vehicular Environments"), an adaptive message delivery protocol which combines geographical and topological information to dynamically adapt its behavior to network conditions. It autonomously selects the most appropriate approach to forward each message based on the input obtained from an adapted implementation of the NeighborHood Discovery Protocol (NHDP) [14]. The design of AVE focusses on the V2I connectivity for cloud services scenario. In this scenario, vehicles send the sensed information as individual and independent messages (bundles), to a cloud service in the Internet, thus requiring messages to be delivered to any available closeby Road Side Unit (RSU).

The AVE approach has been evaluated in a simulated urban scenarios under realistic settings. Realistic propagation models have been used, and urban layouts from the OpenStreetMap [15] database have been included in order to achieve a realistic road layout combined with an accurate building distribution. Results have been analyzed not only to demonstrate its adaptation capabilities under different conditions, but also to detect the areas of possible improvement, highlighting the different trade-offs and how they are addressed by our protocol. Simulations results show that the hybrid and adaptive approach of AVE is able to improve network performance. For example, regarding delivery rate, AVE outperforms DYMO [8] by $10 \%$ in sparse scenarios and outperforms DTN [11] by $10 \%$ in dense scenarios.

The rest of this paper is organized as follows: Section II presents the overall protocol behavior and its implementation, and reviews some of the basic common routing problems, explaining the solution adopted in AVE. Section III evaluates the protocol and discusses the results obtained. Finally, Section IV concludes the paper. 


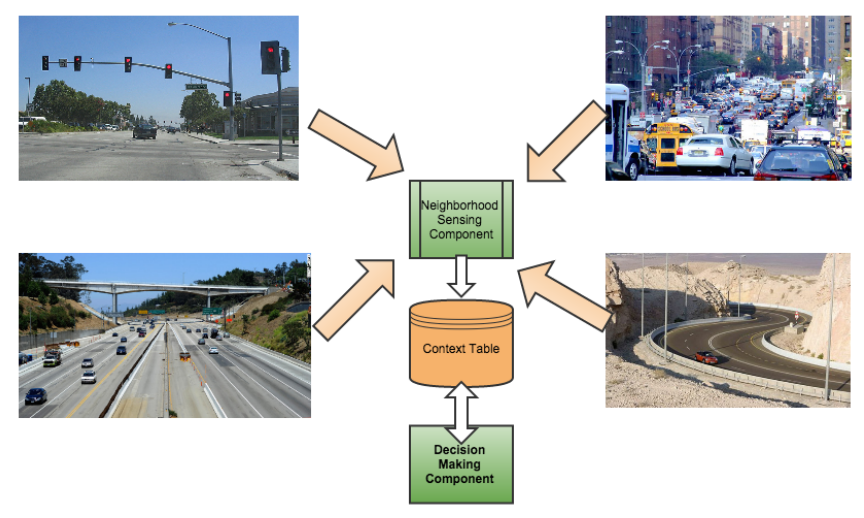

Figure 1: Protocol architecture.

\section{ADAPTIVE ANYCASTING SOLUTION FOR VEHICULAR ENVIRONMENTS}

In anycasting the planned receivers are those specifying interest in the information. In this sense, Our "adaptive Anycasting solution for Vehicular Environments" (AVE) focuses on a scenario where a mobile node wants to send the sensed information to a cloud service in the Internet, thus requiring to deliver individual and independent messages (bundles) to any available close-by Road Side Unit (RSU).

AVE aims to be robust to diverse connectivity possibilities. It autonomously adapts to the current status and context of the network, and reacts by choosing the best forwarding strategy. Figure 1 shows its architecture within four possible contexts: sparse urban, dense urban, sparse highway, and sparse rural.

AVE basically integrates three main elements: a) the neighborhood sensing component, b) the context table, and c) the decision making component. The neighborhood sensing component provides the required input either to update the context table and to make forwarding decisions. In the context table, AVE stores and classifies the neighbors detected, maintains updated location data from one-hop neighbors, as well as information about the stability of the different links, too. The decision making component is in charge of deciding which strategy is the most appropriate to forward the packet according to the network state.

The AVE neighborhood sensing component makes use of periodic beaconing to gain awareness of nearby nodes. It is based on the NeighborHood Discovery Protocol (NHDP) [14] and uses the standard packet format definition described in [16]. The beacon interval can be set arbitrarily, although recommended values are discussed in Section II-C.

Beacons include data about the neighbor topological and geographical state; Figure 2 shows the proposed packet format. It includes relevant information such as, regarding topology: the IP address, the 1-hop neighbors IP addresses, and the link state; regarding location: the current location (coordinates), the current speed/direction, and the final destination (coordinates). Since beacons follow the NHDP packet format definition, their structure could be easily extended according to the rules in [16] to accommodate relevant information.

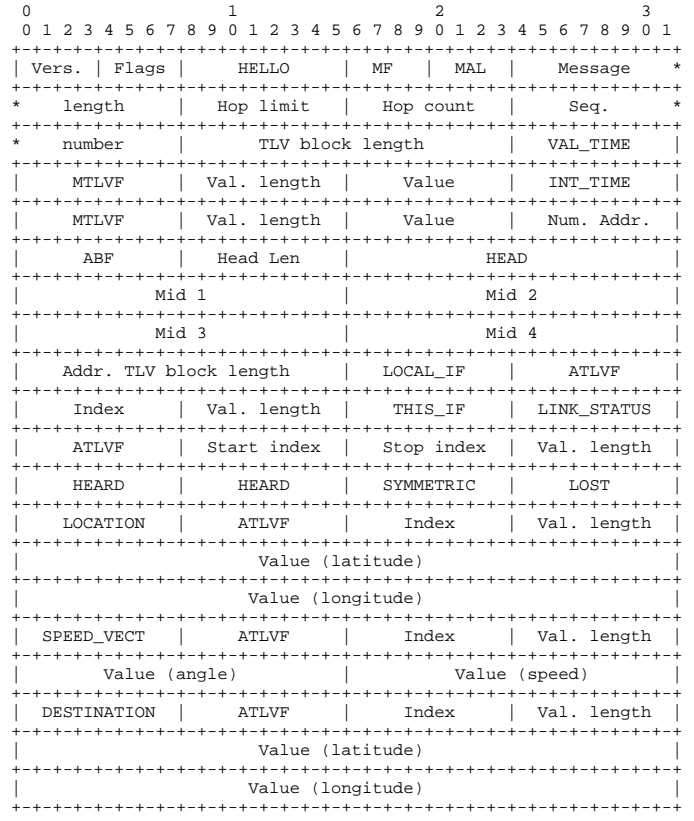

Figure 2: AVE beacons packet format.

\section{A. AVE overall operations}

The decision-making component executes an algorithm that selects, among the available routing strategies, the most adequate one by taking into account the information retrieved about the observed network status maintained in the context table. The decision algorithm is shown in Figure 3.

AVE basically relaxes the delivery delay requirements in a progressive manner to increase delivery reliability. At the same time, it slowly increases the involved memory occupancy in nodes and the signaling overhead. The four strategies used are: local delivery, reactive routing, geographical routing and store-carry-forward delivery.

The local delivery strategy is the fastest and less resource consuming message delivery approach; it is used when the target RSU is only one or two hops away. In case a target RSU is further away, i.e., is not present in the context table, AVE starts a route discovery process. This action takes place only if a beacon from an RSU was received during the last $\mathrm{T}_{M A X}$ seconds.

The $\mathrm{T}_{\text {MAX }}$ parameter prevents the route discovery process from starting. Its value was set taking into account the vehicle speed, the transmission range, and the mean inter-contact rate in a typical city. Studies on the traffic patterns and the contact rates between nodes in the city of Shangai [17] revealed that the inter-contact time of a vehicle with any other vehicle is in the order of several minutes. Nevertheless, a vehicle can still reach an RSU in 5 hops with a delay of about 3 minutes when setting the transmission range to $500 \mathrm{~m}$, considering the case where the route exists, and supposing it travels at the typical maximum city speed of $50 \mathrm{~km} / \mathrm{h}$.

In our tests we set $\mathrm{T}_{M A X}$ to 6 minutes to increase the chances of having a successful route discovery process. The basic reason behind this choice is twofold: (1) we consider that assuming that all vehicles move throughout a city at maximum speed is unrealistic, and (2) propagation in urban scenarios has 


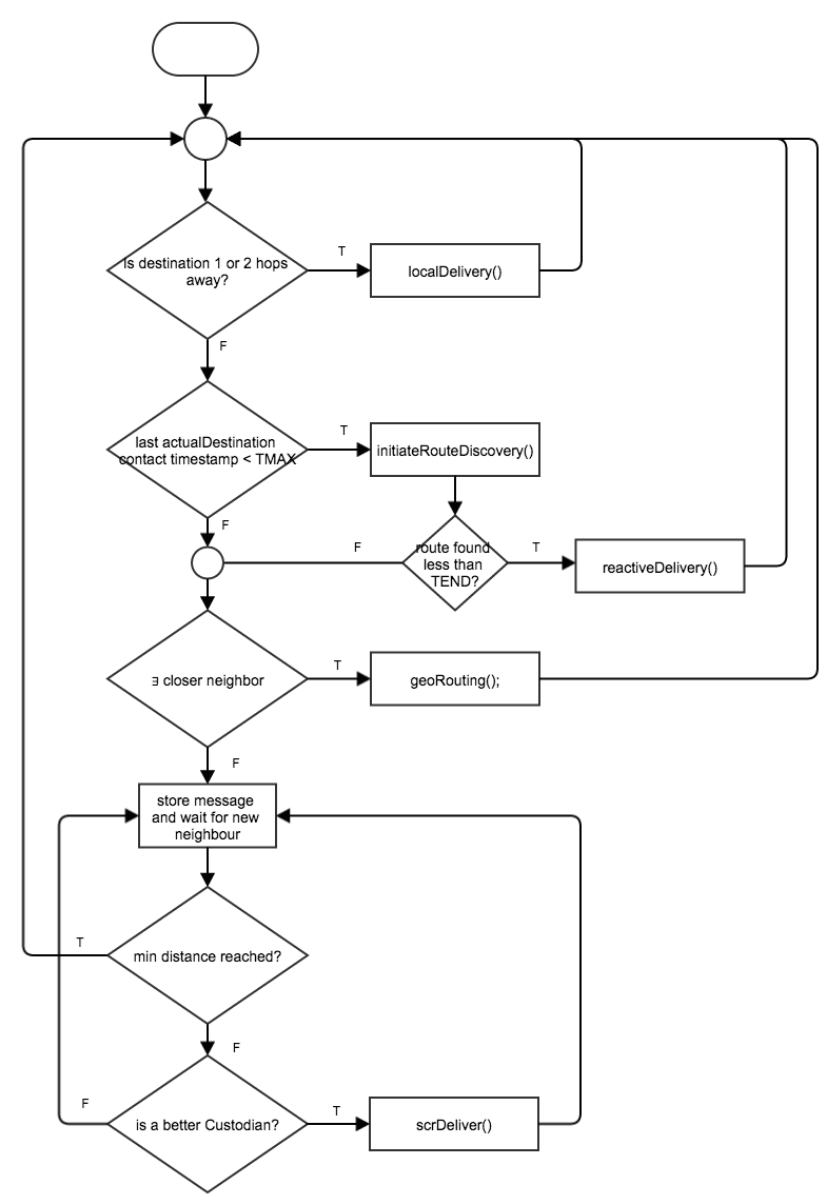

Figure 3: Decision algorithm.

a vast set of interference sources thus making it even more unreliable.

If the route discovery process is unable to find a route within a specified time-out time $\left(\mathrm{T}_{E N D}=10 \mathrm{~s}\right)$, then the decision making component switches to geographical routing, thus relying on the position information of the closest RSU. Eventually, if no neighbors are known, i.e., the node is temporary isolated, the message is stored and carried until another connectivity possibility is available.

\section{B. AVE algorithm details}

The four strategies used, namely: local delivery, reactive routing, geographical routing and store-carry-forward delivery, were selected since they complement each other in a wide range of vehicular scenarios.

The first one, local delivery, is a fast approach that performs well in scenarios with a high RSU density, and where long paths are not expectable. In environments characterized by a low RSU density, the second strategy, based on a reactive protocol takes over to find an optimal path for the message to be delivered, regardless of the roads structure. The third strategy, i.e., the geographical approach, complements the reactive protocol by using a low delay greedy forwarding scheme, although it cannot deal with all road layouts, thereby presenting delays and inaccuracies related to its location service. Finally, the last strategy, the store-carry-and-forward approach, can deal with disconnected networks, covering the remaining network states; it makes predictions about the neighbors location at a future time, using a movement estimation that takes into account their actual position, their speed, and their direction. According to this prediction, we select the best one, that is the node that is supposed to get closer to an RSU sooner, as the message "custodian". This process is repeated whenever a contact between two mobile nodes occurs.

Specific issues had anyway to be solved for each of the selected routing strategies.

Purely MANET-like protocols generally tend to consume most available bandwidth just to get an updated set of routes to destination, or they are too slow and routes become rapidly unavailable. Proactive routing protocols, for example, need a high beacon frequency to keep route information fresh, thus consuming a lot of bandwidth. Additionally, reactive routing protocols need too much time and too much bandwidth to discover long routes. This is why AVE first attempts to make a local delivery, and only if this is not successful it does activate path search. Moreover, we use a bounded reactive approach by reducing the maximum scope of the route search. This allows us i) to avoid long routes that can be possibly broken while the sending process is being carried out, and ii) to avoid wasting network resources with frequent broadcasts storms.

Focussing on the geographic routing protocols, we observe that they are based on a simple idea: look for the closest neighbor, compute the euclidean distance, and forward the packet to it. However, this strategy has two basic drawbacks: i) a greedy strategy does not guarantee finding the right path to the destination, so a recovery protocol must be included, and ii) a location service is needed. As will be shown in Section III, although georouting should be, in theory, equally effective in dense and sparse networks, the combination of these two factors make a reactive routing approach performs better than geographic approaches in terms of packet delivery in more than $10 \%$ of the cases.

In geographical greedy protocols, a recovery process is triggered to find a route to destination when local minimums exist. It is usually based on the right-hand method [18], a method which sends the packet along the border of the network to find a node closer to the destination than the one which started the process. In AVE the recovery procedure is taken care by the reactive protocol or by the store-carry-and-forward approach. When a node is a local minimum for georouting, a route discovery procedure can be started if the restrictions of the algorithms allows (see Section II-C), and the actual route to the destination can be found. If this route cannot be found, the store-carry-forward delivery takes over.

\section{Specific issues}

In this Section we highlight several common problems of communications protocols in vehicular networks, and we show how AVE deals with them. Most of these issues have been addressed by other authors, but they still remain open.

1) The location service: In geographical routing, a component able to translate IP addresses into geographical coordinates is required. Location services are used for this task, 
but packet losses associated to the lack of precision from this service can degrade the overall performance.

AVE uses periodic beaconing as the main source of geographical information, along with the RSU location, which is $a$ priori known and stored in the context table. We have selected beaconing as a location service because it is able to maintain basic location knowledge about the neighbors of the vehicle without requiring other communication technologies (e.g., 3G networks). In this sense, and according for example to [19], [20], [21], choosing an adequate beacon frequency is a critical issue. A high beacon frequency will saturate the network, while a low beacon frequency offers the protocol a poor perception about the network state, thus reducing performance. In AVE we used the recommended value described in the NHDP's RFC document, namely 2 seconds.

2) Neighbor detection: Since wireless networks are deployed over lossy channels, detecting when a node can be a viable neighbor is a difficult task. Wireless links suffer from many communication problems, such as signal fading, interferences or ray reflection. Therefore, nodes cannot be assumed to be stable neighbors immediately after they are detected. Hysteresis is often used to detect when a node is a real neighbor, but this is a slow technique. Thus, it can be deployed in static environments, but it is not able to detect neighbors fast enough in highly dynamic environments.

Instead of using hysteresis, we decided to filter the packet dissemination. Since every beacon contains the location data, we can use this information to exclude as a neighbor those vehicles which are farther away than the theoretical maximum distance that wireless networks can cover. This theoretical maximum distance can be estimated from the local node configuration using the Free Space or the Two-ray ground propagation equations.

3) Loop avoidance: Since AVE involves many independent nodes, network loops may occur unless they are adequately coordinated. The highest probability of loops may appear when the decision algorithm in one node switches to a different strategy from the one selected by, for example, the previous node. In order to avoid this kind of loops, we set a sequential check in the algorithm to control the process, so preventing the selection of the delivery strategy in such an order that could create a loop. Essentially, supposing the routing protocols are numbered sequentially ( 1 - local delivery, 2 - reactive routing, 3 - geographical routing, and 4 - store-carry-forward delivery), if node A selects the routing protocol $m$ to forward the packet to node $\mathrm{B}$, node $\mathrm{B}$ would only choose a protocol $n$ in such a way that $n \leq m$. This behavior is achieved with local delivery, because if node A selects it, this implies that node B is a neighbor of the destination and will use local delivery as well. It is also the case with reactive routing, because discovering a route to destination through node $\mathrm{A}$ implies that node $\mathrm{B}$ knows the route and the packet can also be routed with reactive routing. However, in order to adapt the protocol from dense networks to sparse networks, a transition between geographical routing and the store-carry-and-forward discipline is allowed. To avoid loops in that case, a distance threshold $\left(d_{t h}\right)$ similar to the one implemented in [22] is used in our algorithm. This parameter has a twofold function in our protocol: it does not

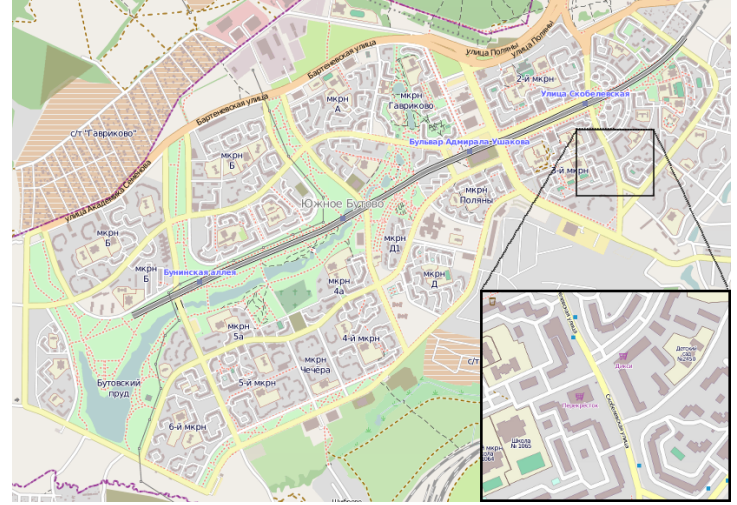

Figure 4: Simulated scenario: General view and detail.

only avoids loops, but it also prevents congestion caused by performing too many route discovery flooding.

\section{Performance evaluation}

In this section we evaluate AVE through simulation using the OMNeT++ [23] simulator and the INETMANET package [24]. We also used different extensions for signal propagation modelling [25], [26] and urban mobility generation [27], [28] to greatly improve its realism.

Vehicular mobility has been defined using VACaMobil [28], a vehicular mobility manager which uses SUMO to achieve a realistic vehicular simulation while maintaining a constant average number of vehicles throughout the whole simulation. We have simulated our network in a real urban scenario: a $12 \mathrm{~km}$ area from the Muscovite suburbs, see Figure 4. In the tests vehicles sent short bursts of messages randomly along their route. Using this traffic pattern we can test the protocol performance for traffic sources in different points of the network, checking where the protocols are able to properly deliver the packets.

We assess the effectiveness of AVE by comparing its performance against a representative protocol for each of the different strategies adopted by AVE, namely with DYMO [8], Greedy georouting [29], and MSDP [30] for DTN routing. The evaluation was made in terms of packet delivery ratio, packet delay, packet losses causes, mean packet hop number, and strategy usage ratio. We also tested the influence of different propagation models.

\section{A. Overall protocol performance}

We first focus on AVE's message delay. Figure 5 compares the performance obtained for message delivery delay for AVE and the other three representative protocols. This scenario can reach two different states depending on the network density. In low densities, only store-carry-and-forward approaches are able to correctly deliver the packets due to network fragmentation. However, under high density networks, location service problems and channel issues makes topological approaches the best option.

AVE obtains an average behavior in the range between the two best options: DYMO and MSDP. The packet delay 


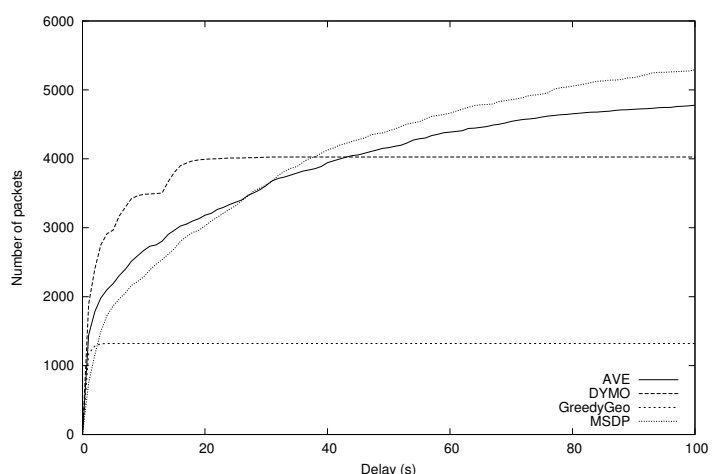

(a) 50 vehicles.

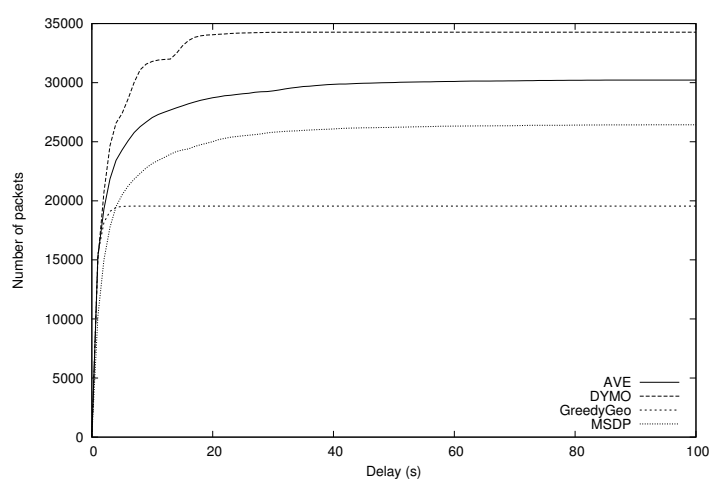

(c) 250 vehicles.

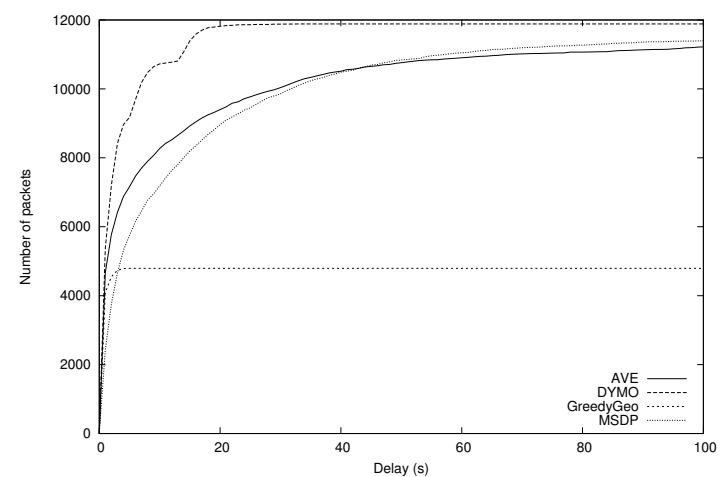

(b) 100 vehicles.

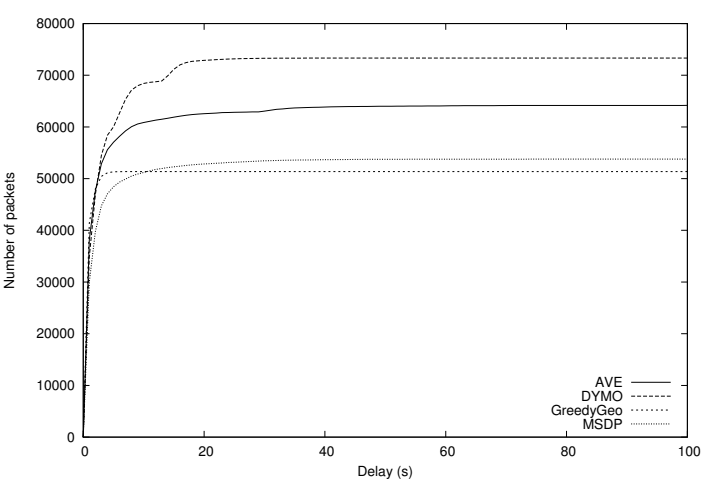

(d) 500 vehicles.

Figure 5: Message delivery delay distribution.

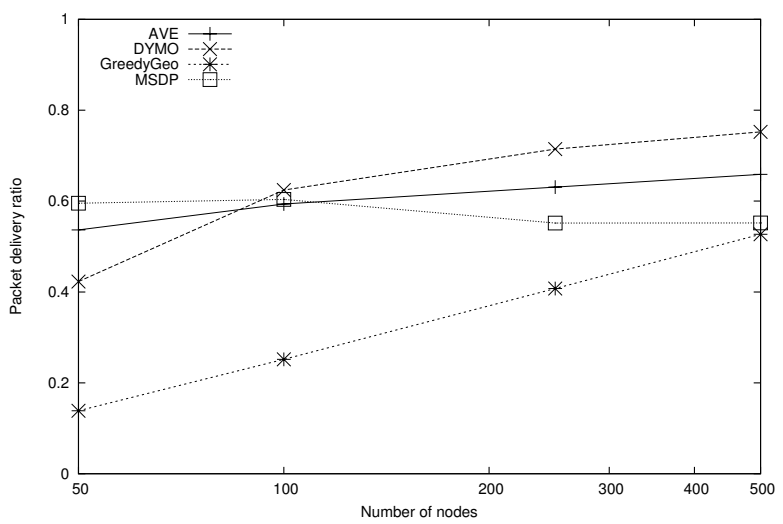

Figure 6: Packet delivery ratio.

increments because AVE is trying to increment the message delivery ratio and to avoid network congestion. On the other hand, AVE outperforms MSDP delay since it can route some packets more quickly using the topological alternatives.

Considering AVE's delivery ratio (see Figure 6), we see that it follows a similar trend. AVE obtains an average behavior in the range between the two best options. This makes AVE the most flexible protocol, achieving a good performance in all the available scenarios. In fact, AVE outperforms DYMO by $10 \%$ in low density scenarios. In dense networks, AVE also outperforms MSDP by $10 \%$ in dense scenarios at the expense of losing only $6 \%$ of data in sparse scenarios.

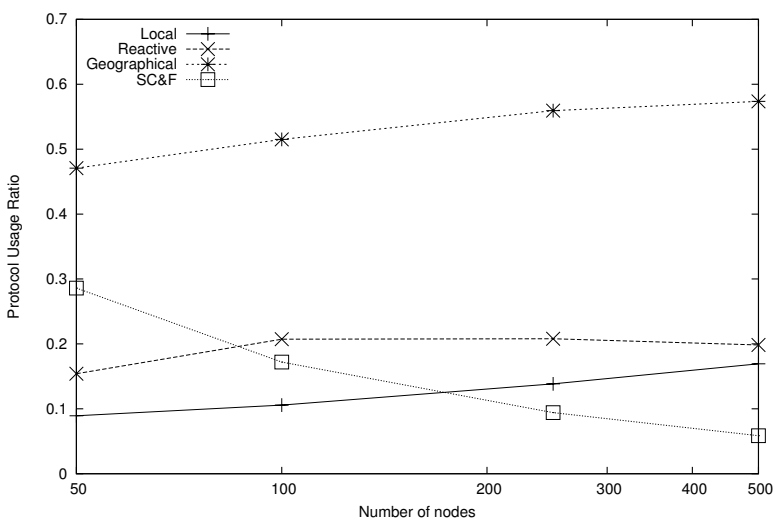

Figure 7: AVE Strategy Usage Ratio.

\section{B. AVE detailed analysis}

In this section we aim at analyzing AVE and exposing its behavior step by step. Due to its modular architecture, AVE can be easily examined and its performance in different network densities can be characterized.

In order to evaluate which protocols are chosen when the next hop is selected, we define the Strategy Usage Ratio (SUR) metric. This metric is defined as the total number of hops travelled by the message using a specific strategy over the total number of hops for the entire simulation. Its mathematical 


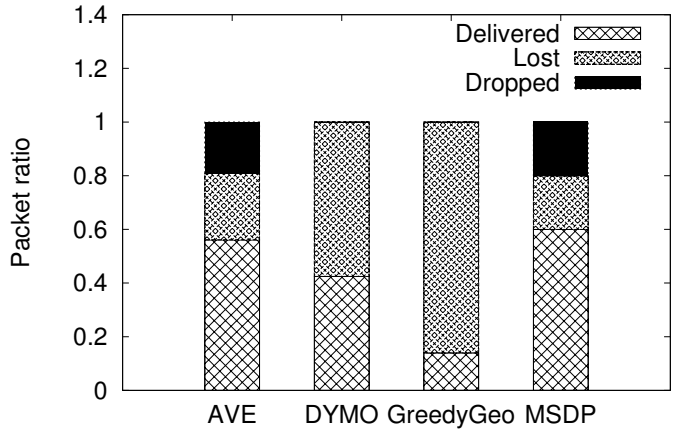

(a) 50 vehicles.

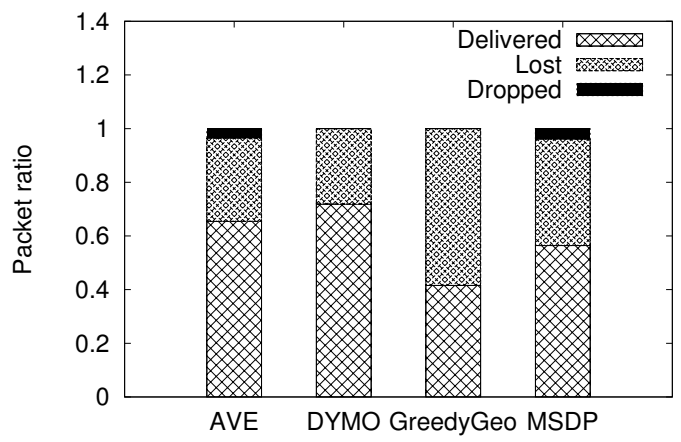

(c) 250 vehicles.

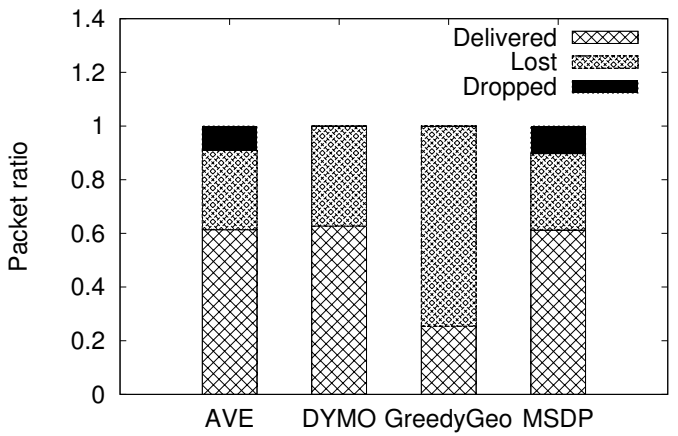

(b) 100 vehicles.

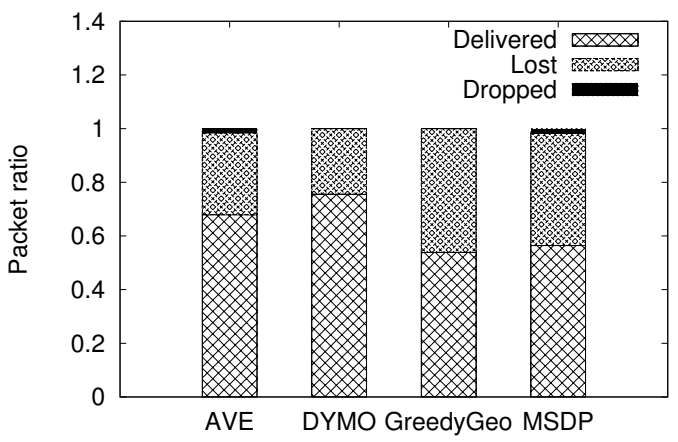

(d) 500 vehicles.

Figure 8: Packet loss causes.

definition can be seen in Equation 1:

$$
S U R(x)=R(x) / \sum_{i=0}^{n} R(i)
$$

where $R(x)$ is the total number of hops travelled by the message using strategy $x$, and $n$ is the total number of strategy items.

Figure 7 shows the SUR value for each strategy under different network densities. We can see how AVE cuts down the usage of store-carry-and-forward strategies by more that $20 \%$ when vehicle density increases. Therefore, the usage of the rest of approaches is incremented by about $10 \%$ of the geographical approaches, and by an additional $10 \%$ of the topological approaches. Moreover, this balance takes place automatically depending on the network disconnection states at different instants of time.

The packet loss causes are shown in Figure 8. There are two kinds of losses: (1) the node ends its trip but it was carrying a packet, and (2) the packet is transmitted to a new node, but it cannot reach its destination. The first group can only be reduced by improving the DTN algorithm. The second group includes both channel losses and location inaccuracy losses. Since the latter is the biggest cause of losses, research must be focused there to improve the final behavior.

This set of figures also shows us an interesting piece of information related to geographical routing. We can see that packet losses in high density networks are not related to network disconnection, as occurs for greedy georouting in sparse networks, nor to a bad custodian selection, because the packet drop rate is low. In this scenario, environmental losses, such as location system inaccuracies and channel fading effects, are more relevant.

\section{Influence of the propagation model on performance}

Finally, in this section we evaluate the performance impact of using a different propagation model. This new propagation model [26] accounts for small moving obstacles that can partly block the signal. Thus, it is a more realistic propagation model for vehicular networks because, in such environments, there are other vehicles, pedestrians, or even trees that can obstruct the signal. Experimentally, it has been measured that the mean propagation distance under this restriction is lower than the one achieved without taking these small obstacles into account.

Figure 9 shows the packet delivery ratio of AVE compared to the performance obtained by each of the representative protocol separately. This propagation model cuts down the overall performance of topological routing protocols, making geographical approaches the best choice for all network states. AVE achieves an average performance in sparse networks that is between two best options and, in addition it outperforms the best option in dense scenarios.

Figure 10 shows a similar trend to the one observed with the previous propagation model. However, in dense networks, we observe that AVE outperforms MSDP latency due to the use of topological approaches. Notice that topological knowledge 


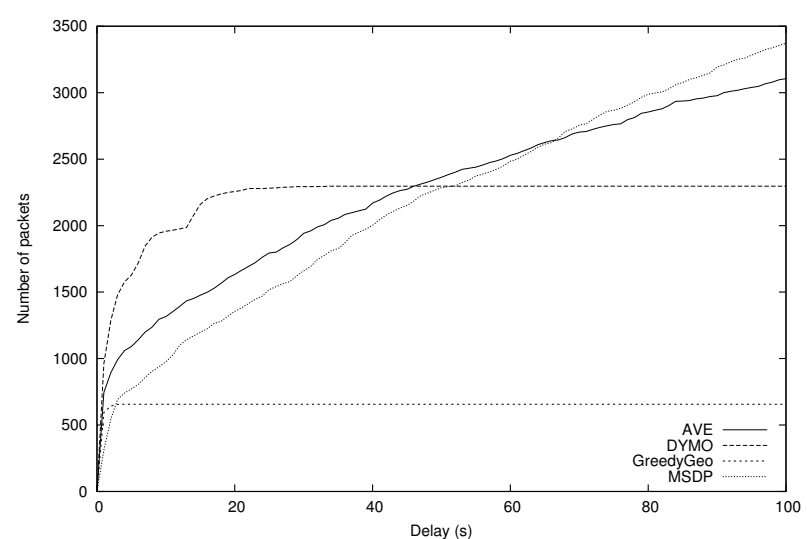

(a) 50 vehicles.

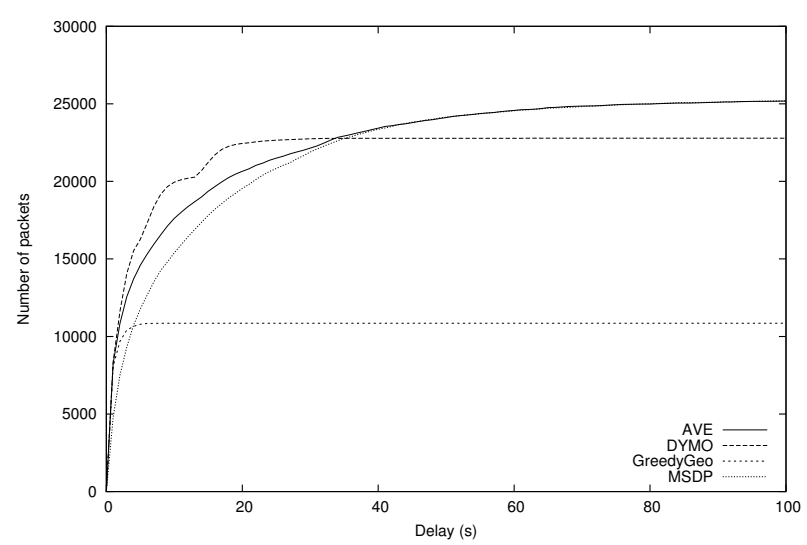

(c) 250 vehicles.

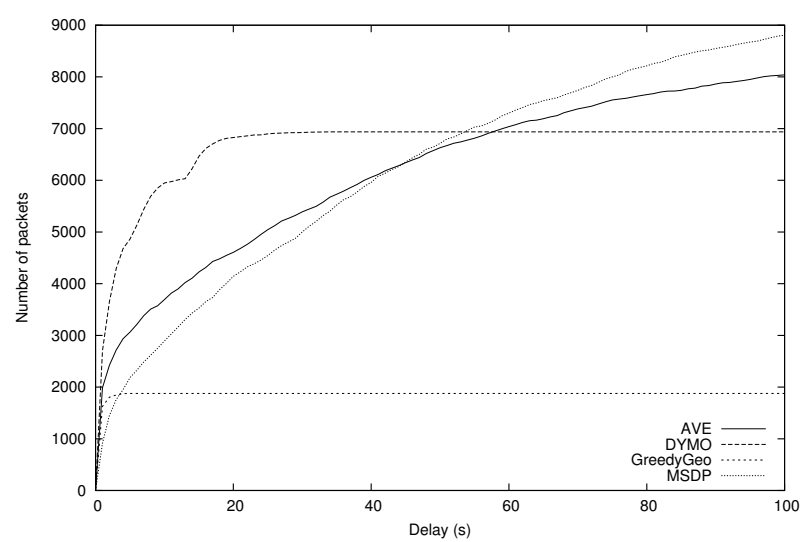

(b) 100 vehicles.

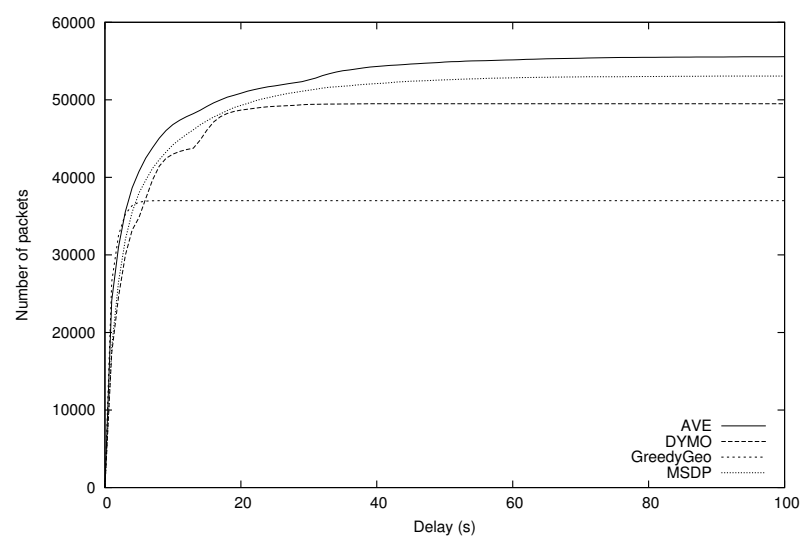

(d) 500 vehicles.

Figure 10: Delay distribution.

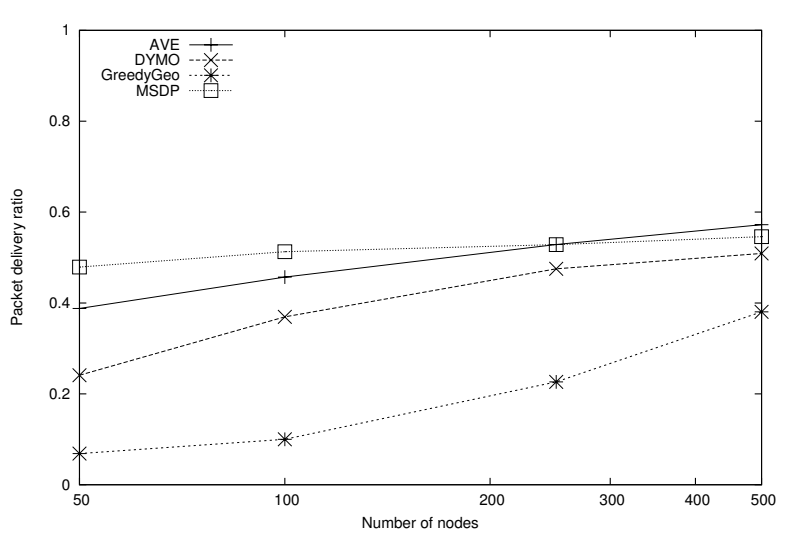

Figure 9: Packet delivery ratio.

is a more precise way to route a packet, since an actual network topology awareness allows to discover the optimal path to route packets to their destination; with this strategy such information is gained through a flooding procedure.

Figure 11 shows the Strategy Usage Ratio when adopting this more realistic environment. In this case, the environment requires a higher flexibility in terms of protocol usage, and AVE doubles its usage of non-DTN approaches in order to improve its delay. In fact, DTN usage is reduced by $25 \%$

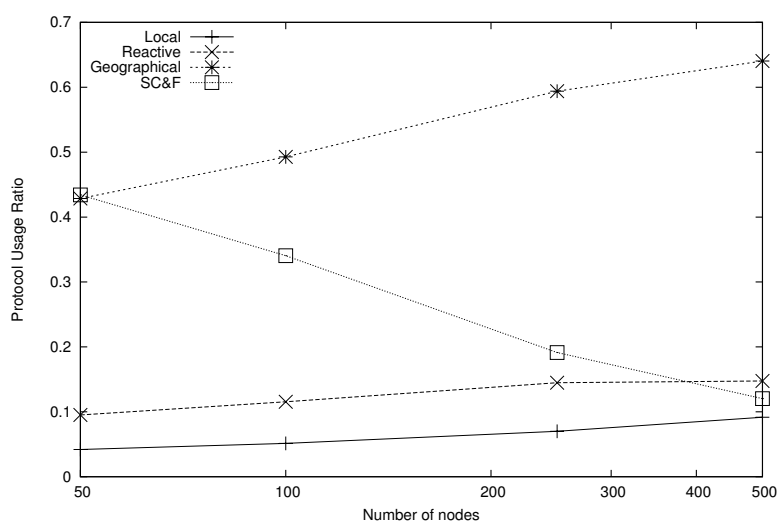

Figure 11: AVE Strategy Usage Ratio.

when comparing the densest against the sparsest scenario; we therefore reduce this result by a factor of two with respect to the case based on the previous propagation model.

\section{CONClusions}

Vehicles traveling can act as mobile sensors and collect a variety of information; but vehicular networks create very varying topologies along time, often including isolated nodes, variable quality links, and variable nodes densities. Such 
highly mutable and heterogeneous context represents a problem for network protocols, which are typically designed for more homogeneous scenarios.

In this paper we presented AVE ("adaptive Anycasting solution for Vehicular Environments"), an adaptive message delivery protocol which combines geographical and topological information to dynamically adapt its behavior to network conditions. It autonomously selects the most appropriate approach to forward each message based on the input obtained from an adapted implementation of the NeighborHood Discovery Protocol (NHDP). In AVE we focussed on a basic scenario: V2I connectivity for cloud services. In this scenario, the vehicles send the sensed information as individual and independent messages (bundles), to a cloud service in the Internet, thus requiring to deliver the messages to any available close-by Road Side Unit (RSU).

The AVE approach was evaluated in simulated urban scenarios under realistic settings. Realistic propagation models were used and urban layouts from the OpenStreetMap database were included in order to achieve a realistic road layout combined with an accurate building distribution. Results were analyzed and showed AVE's adaptation capabilities under different conditions, while also allowing to detect areas of possible improvement, highlighting the different trade-offs and how they are addressed by the protocol. Simulations results showed that the hybrid and adaptive approach of AVE was able to improve network performance. For example, regarding delivery rate, AVE outperformed DYMO by $10 \%$ under sparse scenarios, and outperformed MSDP by $10 \%$ under dense scenarios.

\section{REFERENCES}

[1] W. He, G. Yan, and L. D. Xu, "Developing vehicular data cloud services in the IoT environment," Industrial Informatics, IEEE Transactions on, vol. 10, no. 2, pp. 1587-1595, May 2014.

[2] T. Nolte, I. Shin, M. Behnam, and M. Sjodin, "A synchronization protocol for temporal isolation of software components in vehicula systems," Industrial Informatics, IEEE Transactions on, vol. 5, no. 4, pp. 375-387, Nov 2009.

[3] W. Ni, I. Collings, R. P. Liu, and Z. Chen, "Relay-assisted wireless communication systems in mining vehicle safety applications," Indus trial Informatics, IEEE Transactions on, vol. 10, no. 1, pp. 615-627, Feb 2014.

[4] S. Panichpapiboon and W. Pattara-Atikom, "A review of information dissemination protocols for vehicular ad hoc networks," Communications Surveys Tutorials, IEEE, vol. 14, no. 3, pp. 784-798, Third 2012.

[5] C. Suthaputchakun and Z. Sun, "Routing protocol in intervehicle communication systems: a survey," Communications Magazine, IEEE, vol. 49, no. 12 , pp. 150-156, December 2011.

[6] F. Dressler, H. Hartenstein, O. Altintas, and O. Tonguz, "Inter-vehicle communication: Quo vadis," Communications Magazine, IEEE, vol. 52 no. 6, pp. 170-177, June 2014

[7] X. Tie, A. Venkataramani, and A. Balasubramanian, "R3: Robust replication routing in wireless networks with diverse connectivity characteristics," in Proceedings of the 17th Annual International Conference on Mobile Computing and Networking, ser. MobiCom '11. New York, NY, USA: ACM, 2011, pp. 181-192. [Online]. Available: http://doi.acm.org/10.1145/2030613.2030634

[8] C. Perkins, S. Ratliff, and J. Dowden, "Dynamic MANET on-demand (DYMO) routing," draft-ietf-manet-dymo-26 (work in progress), 2013.

[9] J. Toutouh, J. Garcia-Nieto, and E. Alba, "Intelligent OLSR routing protocol optimization for VANETs," Vehicular Technology, IEEE Transactions on, vol. 61, no. 4, pp. 1884-1894, May 2012.

[10] I. Stojmenovic, "Position-based routing in ad hoc networks," Communications Magazine, IEEE, vol. 40, no. 7, pp. 128-134, 2002
[11] Y. Li, M. Qian, D. Jin, P. Hui, Z. Wang, and S. Chen, "Multiple mobile data offloading through disruption tolerant networks," Mobile Computing, IEEE Transactions on, vol. 13, no. 7, pp. 1579-1596, July 2014.

[12] D. Christmann, R. Gotzhein, S. Siegmund, and F. Wirth, "Realization of try-once-discard in wireless multihop networks," Industrial Informatics, IEEE Transactions on, vol. 10, no. 1, pp. 17-26, Feb 2014.

[13] J. Niu, L. Cheng, Y. Gu, L. Shu, and S. Das, "R3E: Reliable reactive routing enhancement for wireless sensor networks," Industrial Informatics, IEEE Transactions on, vol. 10, no. 1, pp. 784-794, Feb 2014.

[14] T. Clausen, C. Dearlove, and J. Dean, "Mobile Ad Hoc Network (MANET) Neighborhood Discovery Protocol (NHDP)," RFC 6130, 2011.

[15] M. Haklay and P. Weber, "Openstreetmap: User-generated street maps," Pervasive Computing, IEEE, vol. 7, no. 4, pp. 12-18, 2008.

[16] T. Clausen, C. Dearlove, J. Dean, and C. Adjih, "Generalized Mobile Ad Hoc Network (MANET) Packet/Message Format," RFC 5444, 2009.

[17] H. Zhu, L. Fu, G. Xue, Y. Zhu, M. Li, and L. Ni, "Recognizing exponential inter-contact time in vanets," in INFOCOM, 2010 Proceedings IEEE, 2010, pp. 1-5.

[18] P. Bose, P. Morin, I. Stojmenović, and J. Urrutia, "Routing with guaranteed delivery in ad hoc wireless networks," in Proceedings of the 3rd international workshop on Discrete algorithms and methods for mobile computing and communications, ser. DIALM '99. New York, NY, USA: ACM, 1999, pp. 48-55. [Online]. Available: http://doi.acm.org/10.1145/313239.313282

[19] I. Khan and A. Qayyum, "Performance evaluation of aodv and olsr in highly fading vehicular ad hoc network environments," in Multitopic Conference, 2009. INMIC 2009. IEEE 13th International, 2009, pp. 1-

[20] J. Haerri, F. Filali, and C. Bonnet, "Performance comparison of aodv and olsr in vanets urban environments under realistic mobility patterns," in Proc. of 5th IFIP Mediterranean Ad-Hoc Networking Workshop (MedHoc-Net-2006), Lipari, Italy, 2006.

[21] D.-W. Kum, J.-S. Park, Y.-Z. Cho, and B.-Y. Cheon, "Performance evaluation of aodv and dymo routing protocols in manet," in Consumer Communications and Networking Conference (CCNC), 2010 7th IEEE, 2010, pp. 1-2.

[22] P.-C. Cheng, K. C. Lee, M. Gerla, and J. Härri, "Geodtn+nav: Geographic dtn routing with navigator prediction for urban vehicular environments," Mob. Netw. Appl., vol. 15, no. 1, pp. 61-82, Feb. 2010.

[23] A. Varga and R. Hornig, "An overview of the omnet++ simulation environment," in Simutools '08. ICST, Brussels, Belgium, Belgium: ICST, 2008, pp. 1-10.

[24] A. Ariza-Quintana, E. Casilari, and A. T. n. Cabrera, "Implementation of manet routing protocols on omnet++,' in Simutools '08, ser. Simutools '08. ICST, Brussels, Belgium, Belgium: ICST, 2008

[25] A. Kuntz, F. Schmidt-Eisenlohr, O. Graute, H. Hartenstein, and M. Zitterbart, "Introducing probabilistic radio propagation models in omnet++ mobility framework and cross validation check with ns-2," in Simutools'08. ICST, 2008, p. 72

[26] M. Baguena, C. Calafate, J. Cano, and P. Manzoni, “Towards realistic vehicular network simulation models," in Wireless Days (WD), 2012 IFIP, 2012, pp. 1-3.

[27] C. Sommer, R. German, and F. Dressler, "Bidirectionally Coupled Network and Road Traffic Simulation for Improved IVC Analysis," IEEE Transactions on Mobile Computing, vol. 10, no. 1, pp. 3-15, January 2011.

[28] M. Baguena, S. Tornell, A. Torres, C. Calafate, J. Cano, and P. Manzoni, "VACaMobil: VANET Car Mobility Manager for OMNeT++," in Workshop on Smart Communication Protocols and Algorithms (SCPA), 2013 IEEE, 2013, pp. 1077-1081.

[29] B. Karp and H.-T. Kung, "Gpsr: Greedy perimeter stateless routing for wireless networks," in Proceedings of the 6th annual international conference on Mobile computing and networking. ACM, 2000, pp. 243-254.

[30] S. Martinez Tornell, C. Calafate, J.-C. Cano, and P. Manzoni, "A map-based sensor data delivery protocol for vehicular networks," in Ad Hoc Networking Workshop (Med-Hoc-Net), 2012 The 11th Annual Mediterranean, June 2012, pp. 1-8. 